\title{
Aplicações de Game Learning Analytics na abordagem sobre conceitos de matemática
}

Me. Josevandro Barros Nascimento - Universidade Federal Rural De Pernambuco UFRPE- josevandrobarros@yahoo.com.br - https://orcid.org/0000-0001-5077-4555

Dr. Rodrigo Lins Rodrigues - Universidade Federal Rural De Pernambuco - UFRPE rodrigo.linsrodrigues@ufrpe.br - https://orcid.org/0000-0002-3598-5204

Dr. Vladimir Lira Veras Xavier De Andrade - Universidade Federal Rural De Pernambuco - UFRPE - vladimir.andrade@ufrpe.br - http://orcid.org/0000-0002-26792187

Resumo: Este trabalho apresenta um mapeamento sistemático, com a finalidade de entender como estão as pesquisas sobre o tema de Game Learning Analytics - GLA, aplicado com as técnicas de Mineração de Dados Educacionais - (MDE) no contexto dos conteúdos de matemática. A análise destas abordagens fornece contribuições para identificar os conteúdos da matemática que são abordados com GLA, as técnicas MDE utilizadas em games voltados para aprendizagem de matemática, as aplicações learning analytics $(L A)$ na aprendizagem de matemática. Em relação a análise das técnicas, encontramos técnicas como Teste-t, Regressão logística, rede bayesiana, árvore de classificação, regressão linear, correlação, análise de variância e sobrevivência que são métodos que estão sendo mais aplicados à análise de aprendizagem de jogos. Os resultados obtidos com este mapeamento sistemático indicam, uma ausência dos conteúdos de matemática voltados para a aprendizagem dos estudantes do Ensino Fundamental dos Anos Finais. Os resultados desta pesquisa abrangem as principais lacunas de pesquisa envolvendo GLA na educação básica.

Palavras-chave: Mapeamento sistemático, Mineração de Dados Educacionais, Game Learning Analytics.

\section{Applications of Game Learning Analysis in the Approach to Mathematics Concepts}

Abstract. This work presents a systematic mapping, in order to understand how the researches on the theme of Game Learning Analytics - GLA are, applied with the Educational Data Mining techniques - (MDE) in the context of mathematics contents. The analysis of these approaches provides contributions to identify the subjects of mathematics content that are addressed with GLA, the MDE techniques used in games aimed at learning mathematics, the learning analytics (LA) applications in learning mathematics. In the analysis of DEM techniques, we find techniques such as t-test, logistic regression, bayesian network, classification tree, linear regression, correlation, analysis of variance and survival which are methods that are being more applied to game learning analysis. The results obtained with this systematic mapping indicate an absence of mathematics content aimed at learning by students in Final Years Elementary School. The results of this research cover the main research gaps involving GLA in basic education.

Keywords: systematic mapping, Educational Data Mining, Game Learning Analytics

\section{Introdução}

Discutir o ensino de matemática relacionado com o uso dos jogos sérios em um contexto escolar pode ser favorável ao desenvolvimento de uma aprendizagem dinâmica e à construção de reflexões que ajudem na formação de conceitos. Os Jogos Sérios, ou Serious Games, cujos propósitos vão além do entretenimento (Michael e Chen, 2006), têm motivado educadores em todo o mundo a empreender esforços na exploração de seu potencial em sala de aula.

Nesse contexto, as estratégias de Game Learning Analytics - GLA podem ser caracterizadas como promotoras da abordagem experimental do conhecimento V. $19 \mathrm{~N}^{\mathrm{o}} 2$, Dezembro, 2021 RENOTE 
matemático. A análise de aprendizagem de Jogos (Game Learning Analytics) está associada a dois fatores: Learning Analytics (LA), no caso da educação; e Game Analytics (GA), no caso de Jogos Digitais de âmbito geral. A Learning Analytics pode ser definida como a possibilidade de "medição, coleta, análise e produção de relatório de dados sobre os alunos e seus contextos, com fins de entender e otimizar a aprendizagem nos ambientes em que ocorre" (Long \& Siemens, 2011; Siemens, Dawson, \& Lynch, 2013). A partir desse tipo de análise, é possível, por exemplo, a exploração de um conjunto de dados educacionais capazes de elucidar situações relacionadas aos processos de ensino e de aprendizagem, também conhecidos como Educational Data Mining (EDM) - em português, Mineração de Dados Educacionais (MDE) (GRELLER \& DRECHSLER, 2012).

Os objetivos educacionais da Learning Analytics e os artefatos mecânicos e tecnológicos da Game Analytics, quando são combinados, podem ser considerados como Game Learning Analytics (Análise de Aprendizagem de Jogos) - GLA. Os ajustes da Learning Analytics e da Game Analytics podem ser utilizados para a melhoria do processo de aprendizagem por meio do uso dos Jogos Sérios, sendo este associado ao design instrucional (FREIRE, et al 2016). Assim, este estudo pode ser caracterizado como um mapeamento sistemático da literatura, sobre as principais pesquisas que buscam a combinação da Game Learning Analytics (GLA) com as técnicas de Mineração de Dados Educacionais (MDE), com vistas a fornecer mecanismos capazes de identificar, a partir dos games, o conhecimento relativo aos conteúdos de matemática que os estudantes já têm.

\section{Serious Games e Game Learning Analytics}

A categoria de jogos intitulados como Serious Games foi inicialmente desenvolvida para o treinamento em campos de domínio específicos - como na medicina ou na atividade militar, e também aplicados à aprendizagem de idiomas (Baltra, 1990; Del Blanco, et al., 2010). Na atualidade, esse termo é utilizado no desenvolvimento de jogos aplicados à resolução de problemas, entretanto, nas pesquisas acadêmicas, usa-se comumente o termo Jogos Sérios para se referir ao uso de Jogos Digitais para fins educacionais (DJAOUTI, et. al., 2011).

Com o constante interesse nos estudos sobre jogos digitais para o desenvolvimento dos processos de ensino e de aprendizagem, esses artefatos têm se tornado um tema promissor para pesquisas, não apenas no meio acadêmico, mas também em áreas midiáticas e educacionais (MAZIVIERO, 2014). O uso desses instrumentos na educação tem motivado educadores a explorar suas potencialidades nas salas de aula como método pedagógico, como uma forma de motivar as habilidades cognitivas dos estudantes.

Ao desenvolver um jogo sério (Serious Games), deve-se considerar os conceitos educacionais da Learning Analytics e as ferramentas tecnológicas da Game Analytics, cujas combinações podemos classificar como Game Learning Analytcs - GLA (Análise de Aprendizagem de Jogos). Essa combinação pode desenvolver melhoria do uso dos jogos sérios na aprendizagem.

De acordo com Freire, et al., (2016), quando combinamos os elementos da Game Learning Analytcs, podemos analisar o que o jogador (estudante) está fazendo durante a execução do jogo. Favorecendo a investigação em ambientes que relacionam mediação, contribuição, análise dos dados sobre os alunos em seu contexto de análise de jogos com a finalidade de compreender e otimizar a aprendizagem em um espaço. O sistema baseado em Game Learning Analytcs (pode contribuir para coleta de dados associados a vários aspectos que se possa desejar coletar, "podem ser vistos de duas perspectivas diferentes: uma mais técnica sobre o jogo e a infraestrutura do jogo e outra mais focada nos dados RENOTE 
do usuário e experiência." (FREIRE, et al, 2016, p.18). É utilizado para exploração de dados e melhoramento da educação e da ciência da aprendizagem, embora destacando a interação de dados e visualização.

Nesse sentido, relacionamos Game Learning Analytcs com as percepções empíricas da aprendizagem, passando de uma abordagem desenvolvida em teoria para uma abordagem baseada em evidências. Esses dados podem contribuir para a educação, ajudar a esclarecer como se desenvolvem os processos de ensino e de aprendizagem dos jogadores (estudantes), relacionados ao conteúdo de matemática.

\section{Método}

O protocolo adotado para esta pesquisa segue as orientações de Moher e Shekelle (2015), que apresentam as definições fundamentais para o desenvolvimento de um mapeamento sistemático da literatura, ou revisão de escopo (scoping review), em que apontam que nesse tipo de pesquisa não é necessário responder com profundidade a questões específicas, mas trazer uma visão ampla do objeto de estudo de uma determinada área.

\subsection{Definindo a Questão de Pesquisa}

Para o mapeamento sistemático realizado, foi ponto de partida a questão: Como a combinação da Game Learning Analytics (GLA) com as técnicas de Mineração de Dados Educacionais (MDE) pode ajudar a identificar, a partir dos games, os conhecimentos, já aprendidos por estudantes, relativos aos conteúdos de matemática?

Dessa pergunta, foram desmembradas três questões secundárias:

Q1 - Quais conteúdos da matemática são abordados nesses estudos?

Q2 - Quais técnicas de mineração de dados educacionais (MDE) podem ser utilizadas em games voltados para aprendizagem de conceitos relativos à matemática?

Q3 - Quais são os objetivos da aplicação da learning analytics (LA) na aprendizagem de matemática com games?

As respostas a tais perguntas ensejam os objetivos do trabalho.

\subsection{Busca e Seleção dos Estudos}

Com vistas a responder às questões de pesquisa apresentadas, foram consultadas para a constituição do corpus a IEEE Xplore, ACM Digital Library, ScienceDirect, SpringerLink, Taylor \& Francis Online, dentre muitas outras bibliotecas digitais. Nessas bibliotecas digitais foram aplicadas as palavras-chave que melhor remetiam para as questões norteadoras do mapeamento sistemático dessa pesquisa, resultando nas strings de busca: ("Analytics" OR "Learning Analytics" OR "Educational Data Mining") AND ("Game" OR "Serious Games") AND ("Mathematic Game" OR "Math Game"), conforme o fluxograma abaixo:

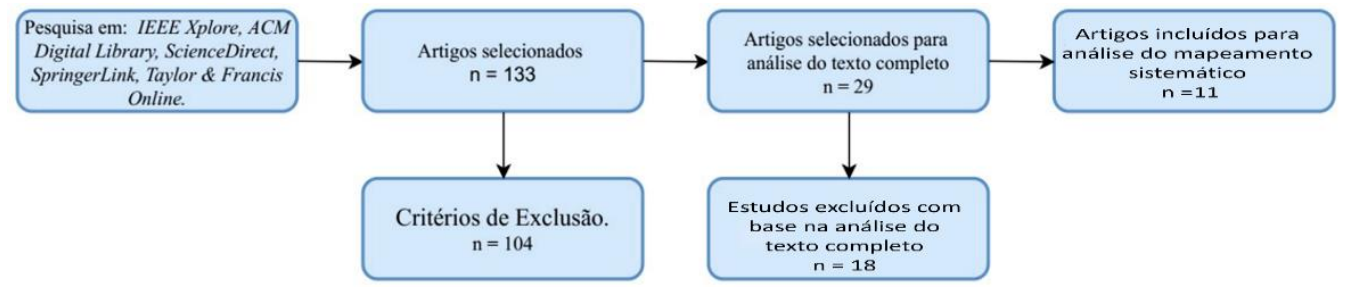

Figura 1. Fluxograma do mapeamento sistemático

Fonte: Elaborada pelas autoras (2021) 
Com os critérios de buscas definidos, foram localizados inicialmente 133 (cento e trinta e três) trabalhos em plataformas de bibliotecas digitais. A este resultado primário, aplicaram-se os critérios de exclusão e inclusão, conforme as ideias de Dermeval (2019). Os autores definem em suas considerações metodológicas sobre as aplicações de critérios de exclusão em mapeamentos sistemáticos que para excluir um artigo basta que este se enquadre em um dos critérios de exclusão definidos pelo pesquisador; enquanto para a inclusão de um artigo no grupo que será submetido à análise final, devem ser satisfeitos todos os critérios definidos. Assim, apresentamos na Tabela 1 os critérios de exclusão e inclusão adotados.

Tabela 1 - Critérios de exclusão e inclusão

\begin{tabular}{|l|l|}
\hline Exclusão. & $\begin{array}{l}\text { CE01 - Artigos de revisão sistemática ou mapeamento sistemático; } \\
\text { CE02 - Trabalhos duplicados. }\end{array}$ \\
\hline Inclusão. & $\begin{array}{l}\text { CI01- artigos que no resumo tragam Analytic }+ \text { Games }+ \text { Math; } \\
\text { CI02- Artigos de fonte primárias; } \\
\text { CI03 - Artigos que tenha Analytic }+ \text { Games }+ \text { conteúdo de } \\
\text { matemática + atividades de matemática }\end{array}$ \\
\hline
\end{tabular}

Com base nos critérios de exclusão CE01 e CE02, foram excluídos 104 (cento e quatro) artigos constantes dos resultados preliminares de busca, ficando apenas 29 (vinte e nove) artigos selecionados para análise completa dos textos. Com base na análise dos textos completos, foram excluídos outros 18 (dezoito) artigos, por não estarem adequados aos critérios de inclusão. Dessa forma foram selecionados 11 (onze) artigos para compor análise final do mapeamento sistemático, que subsidiou as análises e interpretações dos resultados ora apresentados.

\section{Análise e Interpretação dos Resultados}

Com os 11 (onze) artigos selecionados, foram realizadas análises do escopo do texto completo, considerando os objetivos, fundamentação teórica, metodologias, resultados/discussões e conclusões para o aprofundamento do mapeamento sistemático da literatura. Nessa fase foram avaliados os artigos e finalmente incluído para análise e interpretação dos resultados para responder à questão de pesquisa, onde foram elaboradas estratégias para categorização, conforme Dermeval (2019). Na sequência, apresentamos a síntese dos trabalhos, relacionado-os com os questionamentos expostos na seção 4.1.

4.1 Q1 - Quais conteúdos da matemática são abordados nesses estudos?

A proposta dessa questão de pesquisa a de reunir as evidências presentes na literatura científica e as abordagens relacionadas com Base Nacional Comum Curricular - BNCC que denotem quais conteúdos de matemática estão sendo abordados nos estudos da Game Learning Analytic (GLA) e da Mineração de dados (MDE), conforme a figura 2 abaixo. 


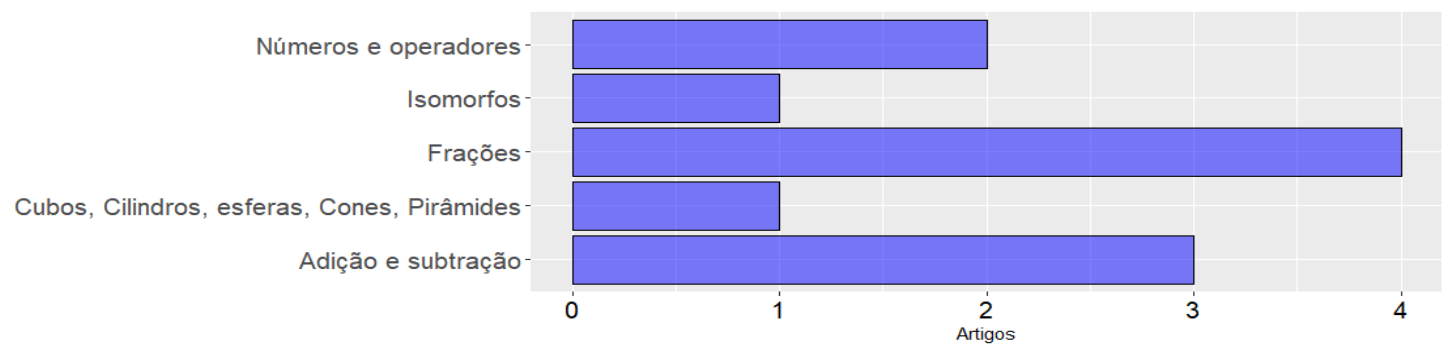

Figura 2 - Conteúdos da matemática.

Fonte: Elaborada pelas autoras (2021)

Dos 11 (onze) artigos analisados, três pesquisas, de KIM, Paul, (2012), Akintunde (2020) e KIM, Bugeun (2018), abordaram os conceitos da unidade temática "números e operadores", que favorecem a investigações em ambientes de ensino e a aprendizagem em que desperta motivações e provoca curiosidades. Nessa unidade temática, por meio de jogos, são abordados os conteúdos de conhecimentos da adição e da subtração. De acordo com a Base Nacional Comum Curricular - BNCC (BRASIL, 2018), os objetos de conhecimento da adição e da subtração desenvolvem nos estudantes habilidades e percepções da compreensão que deve ser aplicada e utilizada no cálculo mental ou escrito. Quanto ao uso dos jogos aplicados aos objetos de conhecimento da adição e subtração, os jogos propiciam um ambiente contextualizado e significativo.

Apenas uma pesquisa, de Ruipérez-Valiente (2020), aborda as unidades temáticas da geometria por meio de jogos, tratando dos conhecimentos sobre cubos, cilindros, esferas, cones e pirâmides. Para a BNCC (2018), é importante "Reconhecer, nomear e comparar figuras geométricas espaciais (cubo, bloco retangular, pirâmide, cone, cilindro e esfera), relacionando-as com objetos do mundo físico" (BRASIL-BNCC, 2018, pág. 283). Assim, compreendemos que as unidades temáticas da geometria atrelados aos jogos podem trazer resultados esperados de uma aprendizagem significativa.

Foram encontradas também as pesquisas de Ninaus et al. (2017), Peddycord-Liu (2017 e 2018) e Lindstedt (2019) que abordam as unidades temáticas de números, no entanto são aplicados ao conteúdo de frações. Por meio de jogos, os autores abordam a estratégias e os conceitos de frações. É pensando nessa aplicação dos objetos de conhecimento relacionando-os com o uso dos jogos em matemática que os autores relacionam os números racionais em um percurso no desenvolvimento de outros conteúdos matemáticos.

O gráfico também nos indica que foi encontrada uma pesquisa de Dou (2010) em que o objeto de conhecimento abordado é o isomorfo, corroborando com os conceitos da unidade temática Álgebra da BNCC. Quando é abordado o conceito do isomorfo atrelado aos jogos, torna-se uma estratégia ou uma metodologia didática que desenvolve nos estudantes a percepção da construção dos conceitos baseado na resolução de problemas aplicados com os jogos, favorecendo, assim, para uma aprendizagem.

Quanto às pesquisas de Alexandrovsky (2021) e De Chambrier (2021), que focalizam o conceito de números e operadores, o qual está associado a solucionar e desenvolver problemas com números racionais positivos, na concepção decimal, com operadores envolvendo as quatro operações fundamentais da aritmética que é descrito com os jogos e que tem o propósito melhor aprendizagem dos estudantes. Nessa ideia é desenvolvida a resolução de problema com a utilização dos jogos aplicando os conceitos de números e operadores facilitando o ensino e aprendizagem dos conteúdos matemáticos. 
Diante do que foi apresentado, entendemos que os conteúdos da matemática são pouco aplicados na utilização de Game Learning Analytic e Mineração de Dados para educação básica.

4.2 Q2 - Quais técnicas de Mineração de Dados Educacionais (MDE) podem ser utilizadas em games voltados para aprendizagem de conceitos relativos à matemática?

Para discutir as respostas encontradas para tal questão, foram identificadas, nos 11 (onze) artigos científicos, 16 técnicas de MDE. Nessas publicações, alguns autores estudam uma ou mais técnicas de MDE, que apresentamos categorizadas a seguir na figura 2.

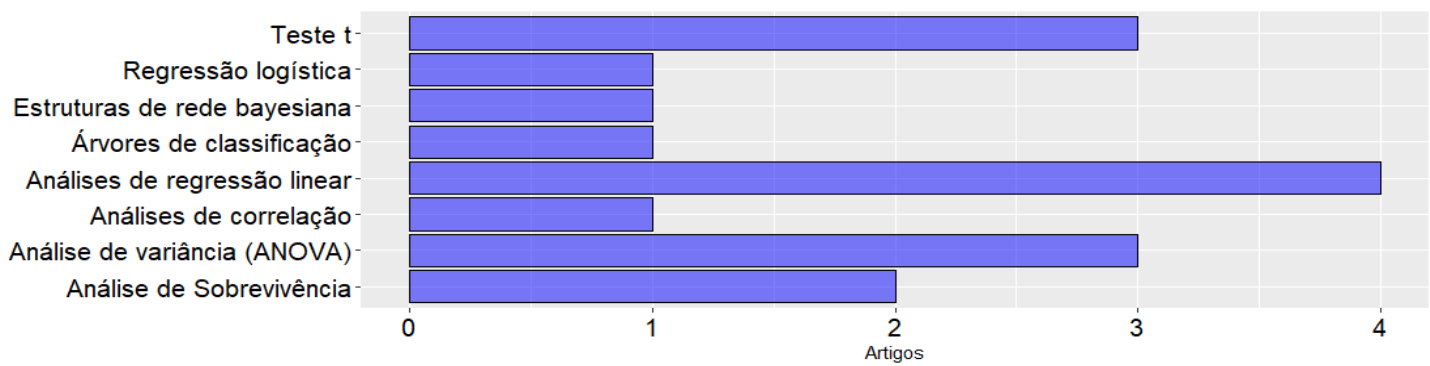

Figura 2 -Técnicas de Mineração de Dados Educacionais (EDM).

Fonte: Elaborada pelas autoras (2021)

$\mathrm{Na}$ análise da técnica de MDE, encontramos dois trabalhos, de KIM, Bugeun (2018) e Alexandrovsky (2021), que se utilizam das técnicas de análise de sobrevivência para o registro e coleta dos dados enquanto os estudantes jogam o game. Ao se utilizarem da análise de sobrevivência aplicada com os jogos em suas pesquisas, os autores deixam evidente que os resultados desta facilitam para analisar o desempenho da aprendizagem e o comportamento dos jogadores (estudantes) após o jogo.

Nas publicações de DOU (2010), KIM, Paul (2012), Ninaus et al. (2017) os autores partem de uma perspectiva do uso das técnicas de MDE, com a utilização da Análise da Variância (ANOVA) para medir a aprendizagem dos estudantes durante a execução do game. Esse método contribui para analisar as médias de problemas que foram resolvidos na interação dos jogadores (estudantes) com os jogos, através de grupos, ou a interação entre duas ou mais variáveis aplicadas no desenvolvimento das etapas do jogo. O gráfico menciona apenas um artigo que trata da técnica de análise de correlação, a qual Ninaus et al. (2017) aplica em sua pesquisa com o uso do game para avaliar as relações positivas entre a comparação do conteúdo de fração com a aprendizagem dos estudantes. $\mathrm{O}$ método de análise de correlação fornece evidências para avaliar o grau de aprendizagem dos estudantes associando a velocidade da execução do jogo e as comparações de conteúdos abordados durante o jogo. Portanto, a análise de correlação é aplicada para avaliar a comparação entre conteúdos e aprendizagem.

Nas pesquisas de Peddycord-Liu (2017), Akintunde (2020), De Chambrier (2021) e Lindstedt (2019), encontra-se o método estatístico da análise de regressão linear, utilizado para descobrir qual a progressão da aprendizagem a partir dos dados coletados no game. Esse método possibilita informar o sequenciamento da aprendizagem dos estudantes mediante a coleta dos dados. Além disso, a análise ajuda a descobrir resultados educacionais importantes na aprendizagem dos estudantes contribuindo para uma flexibilidade da ordem dos conteúdos matemáticos para os estudantes jogarem.

$\mathrm{Na}$ produção de Akintunde (2020), além do uso do método estatístico da regressão linear, aplica-se o método da árvore de classificação na verificação da 
aprendizagem por meio do game. As árvores de classificação são um método preditivo que valida o número de jogadas dentro dos games.

O gráfico indica a ocorrência da técnica de Regressão logística, apenas em uma pesquisa, de Peddycord-Liu (2018), e está empregada no game para compreender os diferentes campos do conhecimento dos estudantes enquanto jogam. Essa técnica compreende representações estatísticas/matemáticas com o desempenho do jogador em uma atividade repetitiva durante a execução do game. O método de Regressão logística está associado à curva de aprendizagem, é utilizado para avaliar o quanto um jogo educacional apoia a aprendizagem. Além do método estatístico da análise de regressão linear aplicado por Peddycord-Liu (2017), sua pesquisa está associada também à estrutura/análise de rede Bayssiana, usada para analisar as habilidades e os desempenhos de cada estudante durante a execução do jogo.

Nas pesquisas de Ruipérez-Valiente (2020), KIM, Bugeun (2018) e Ninaus et al. (2017), a fim de analisar as diferenças nos quantitativos das variáveis obtidas pela execução do game, foi aplicado o Teste $t$, que revela diferenças entre os comportamentos dos estudantes por meio de suas jogadas. Este método comprova a possibilidade de a análise de aprendizagem ser utilizada para observação pedagógica e proporciona o feedback sobre o comportamento do estudante na execução do jogo. $\mathrm{O}$ teste $\mathrm{t}$, contribui para analisar os padrões do game e inspeciona a diferença entre características da aprendizagem dos estudantes.

Consideramos que essas técnicas/métodos podem ser aplicadas no desenvolvimento da análise de aprendizagem de jogos aplicados com os conteúdos de matemática. Nesse sentido, relacionando as técnicas de Mineração de Dados Educacionais (MDE), compreendemos que é importante para feedback do professor o que o estudante está aprendendo durante a execução do jogo.

4.3 Q3 - Quais são os objetivos da aplicação learning analytics (LA) na aprendizagem de matemática com games?

Apesar de alguns autores coincidirem nos conteúdos abordados e nas técnicas de coletas de dados empregadas durante a execução dos games, divergem na aplicação do LA na aprendizagem de matemática, por isso procurou-se identificar quais estudos os autores trazem com o uso do LA na aprendizagem de matemática com os games. DOU (2010) assume como objetivo de sua pesquisa saber como os estudantes puderam desenvolver os conceitos de isomorfo e analisar quais estratégias foram empregadas durante a execução do game. Já os objetivos das pesquisas com o LA de KIM, Paul (2012) incluem resolver uma sequência de problemas matemáticos, por meio do game, sem intervenção ou instrução por outras pessoas.

As pesquisas de Peddycord-Liu (2018) e Akintunde (2020) observam e avaliam a sequência didática de conteúdo em currículo de matemática. Ninaus et al. (2017), avalia os conhecimentos dos estudantes relacionando-os com o conteúdo de fração e maneiras de fazer matemática, além de possibilitar a resolução de problemas. KIM, Bugeun (2018) relaciona a matemática a um aplicativo de aprendizagem móvel desenvolvido tendo como público-alvo as crianças da Educação Infantil.

Diante dos estudos realizados por Peddycord-Liu (2017 e 2018), em que o autor analisa e modela a aprendizagem, por meio de games com atividade de grande escala, que viabiliza ao estudante reforçar as habilidades matemáticas, por meio de resoluções de problemas, e mostrando aos designers um feedback para melhorar os conteúdos do jogo, dando suporte à aprendizagem. Constatamos em nossos estudos que Lindstedt (2019) avalia, em sua pesquisa, por meio da implementação da interface do game, como esta 
influência na eficácia da experiência do estudante com os conteúdos de matemática. Os estudos de Alexandrovsky (2021) contribuíram com as investigações do desenvolvimento da mecânica do jogo com a implementação dos conceitos de matemática para analisar a aprendizagem dos estudantes durante cada fase do jogo. De Chambrier (2021) desenvolveu reflexões sobre a análise de aprendizagem, através dos games no qual desenvolva habilidade matemáticas.

$\mathrm{Na}$ aplicação learning Analytics, observa-se que os conteúdos e as técnicas convergem entre alguns autores, e estão associados a um contexto dinâmico que favorece ao desenvolvimento de uma aprendizagem significativa por meio dos games. Assim sendo, os objetivos aplicados ao games parecem ser um instrumento favorável para aplicação dos conteúdos de matemática. Nesse sentido, o uso learning analytics ajuda a esclarecer como se desenvolvem os processos de ensino e de aprendizagem dos jogadores.

\section{Considerações Finais}

Neste mapeamento sistemático, foram analisados onze estudos de fontes primárias que utilizaram Game Learning Analytic (GLA) e Mineração de Dados (MDE) para observar a aprendizagem de estudantes acerca de conteúdos da matemática. Essas pesquisas foram publicadas em bibliotecas e plataformas digitais de grande relevância científica e apresentavam limitações nos conteúdos de matemática, técnicas de mineração de dados educacionais e a aplicação learning analytics (LA).

$\mathrm{O}$ uso de estratégias de Game Learning Analytics, que tem como foco os jogos sérios, carece de mais estudos que busquem relacionar a abordagem experimental ao conhecimento matemático, favorecendo a investigação em ambientes de ensino e aprendizagem, bem como preencher lacunas relacionadas ao conteúdo de matemática. São poucas as publicações encontradas que relacionam Game Learning Analytic (GLA) e Mineração de dados (MDE) para os conteúdos de matemática. No corpus selecionado, foram analisados estudos que abordam os conteúdos de matemática de acordo com a BNCC (2018), para Ensino Fundamental dos Anos Finais, e não foram identificados trabalhos que detalhem, com profundidade quais técnicas de mineração de dados educacionais (MDE) são utilizadas para análise da aprendizagem com os jogos. A par disso, espera-se desenvolver pesquisas futuras que contribuam para os conteúdos de matemática para o Ensino Fundamental dos Anos Finais.

\section{Agradecimentos}

O presente trabalho foi realizado com apoio da Coordenação de Aperfeiçoamento de Pessoal de Nível Superior - Brasil (CAPES) - processo nº 88887.610260/2021-00

\section{Referências}

AKINTUNDE, Ruth Okoilu; SHABRINA, Preya; CATETE, Veronica; BARNES, Tiffany; LYNCH, Collin; RUTHERFORD, Teomara. Data-informed Curriculum Sequences for a Curriculum-Integrated Game. Acm Digital Library, [s. l], p. 635-644, 23 mar. 2020. Disponível em: https://dl.acm.org/doi/10.1145/3375462.3375530. Acesso em: 14 maio 2021.

ALEXANDROVSKY, Dmitry; FRIEHS, Maximilian Achim; GRITTNER, Jendrik; PUTZE, Susanne; BIRK, Max V.; MALAKA, Rainer; MANDRYK, Regan L. Serious Snacking: a survival analysis of how snacking mechanics affect attrition in a mobile serious game. Proceedings Of The 2021 Chi Conference On Human Factors In 
Computing Systems, [S.L.], p. 1-18, 6 maio 2021. ACM. http://dx.doi.org/10.1145/3411764.3445689.

BALTRA, Armando. Language Learning through Computer Adventure Games. Simulation \& Gaming, [S.L.], v. 21, n. 4, p. 445-452, dez. 1990. SAGE Publications. http://dx.doi.org/10.1177/104687819002100408.

BELLOTTI, Francesco; KAPRALOS, Bill; LEE, Kiju; MORENO-GER, Pablo; BERTA, Riccardo. Assessment in and of Serious Games: an overview. Advances In HumanComputer Interaction, [S.L.], v. 2013, p. 1-11, 2013. Hindawi Limited. http://dx.doi.org/10.1155/2013/13686

BRASIL. Base Nacional Comum Curricular. Brasília: MEC, 2018. Disponível em: http://basenacionalcomum.mec.gov.br/images/BNCC_EI_EF_110518_versaofinal_site. pdf. Acesso em: 10 mar. 2021.

CHAMBRIER, Anne-Françoise de; BAYE, Ariane; TINNES-VIGNE, Mélanie; TAZOUTI, Youssef; VLASSIS, Joëlle; PONCELET, Débora; GIAUQUE, Nadine; FAGNANT, Annick; LUXEMBOURGER, Christophe; AUQUIÈRE, Amélie. Enhancing children's numerical skills through a play-based intervention at kindergarten and at home: a quasi-experimental study. Early Childhood Research Quarterly, [S.L.], v. 54, p. 164-178, 2021. Elsevier BV. http://dx.doi.org/10.1016/j.ecresq.2020.09.003.

COLLETT, David. Modelling survival data in medical research. United Kingdom: Chapman And Hall/Crc, 2014. 548 p.

DOU, Wenwen; ZIEMKIEWICZ, Caroline; HARRISON, Lane; JEONG, Dong Hyun; RYAN, Roxanne; RIBARSKY, William; WANG, Xiaoyu; CHANG, Remco. Comparing different levels of interaction constraints for deriving visual problem isomorphs. 2010 Ieee Symposium On Visual Analytics Science And Technology, [S.L.], p. 195-202., out. 2010. IEEE. http://dx.doi.org/10.1109/vast.2010.5653599.

DEL BLANCO, Á., MARCHIORI, E. J., \& Fernández-Manjón, B. Adventure Games and Language Learning. In First International Workshop on Technological Innovation for Specialized Linguistic Domains: Theoretical and Methodological Perspectives 2010.

DJAOUTI, D., ALVAREZ, J., JESSEL, J.-P., \& Rampnoux, O. Origins of Serious Games. In Serious Games and Edutainment Applications. London: Springer London. 2011.

DERMEVAL, Diego; COELHO, Jorge AP de M. BITTENCOURT, Ig Ibert. Mapeamento sistemático e revisão sistemática da literatura em informática na educação. JAQUES, Patrícia Augustin; PIMENTEL, Mariano; SIQUEIRA; Sean; BITTENCOURT, Ig. (Org.) Metodologia de Pesquisa em Informática na Educação: Abordagem Quantitativa de Pesquisa. Porto Alegre: SBC, 2019.

FREIRE, Manuel; SERRANO-LAGUNA, Ángel; IGLESIAS, Borja Manero; MARTÍNEZ-ORTIZ, Iván; MORENO-GER, Pablo; FERNÁNDEZ-MANJÓN, Baltasar. Game Learning Analytics: learning analytics for serious games. Learning, Design, And Technology, [S.L.], p. 1-29, 2016. Springer International Publishing. http://dx.doi.org/10.1007/978-3-319-17727-4 21-1.

GRELLER, Wolfgang; DRACHSLER, Hendrik. Translating learning into numbers: A generic framework for learning analytics. Journal of Educational Technology \& Society, v. 15, n. 3, p. 42-57, 2012. 
KIM, Paul; BUCKNER, Elizabeth; KIM, Hyunkyung; MAKANY, Tamas; TALEJA, Neha; PARIKH, Vallabhi. A comparative analysis of a game-based mobile learning model in low-socioeconomic communities of India. International Journal Of Educational Development, [S.L.], v. 32, n. 2, p. 329-340, mar. 2012. Elsevier BV. http://dx.doi.org/10.1016/j.ijedudev.2011.05.008.

KIM, Bugeun; RHIM, Jungwook; RHO, Jihyun; HWANG, Taehyun; LEE, Gunho; GWEON, Gahgene. "I'll do it!": examining the relationship between locus of control and math game retention for preschoolers. Proceedings Of The 8Th International Conference On Learning Analytics And Knowledge, [S.L.], v. 5, n. 8, p. 290-294, 7 mar. 2018. ACM. http://dx.doi.org/10.1145/3170358.3170368.

LONG, Phil.; SIEMENS, George. Penetrating the Fog: Analytics in Learning and Education. 2011. Disponível em: https://er.educause.edu/articles/2011/9/penetratingthe-fog-analytics-in-learning-and-education. Acesso em: 05 set. 2020.

LINDSTEDT, Antero; KIILI, Kristian. Effect of Whole-Body Movement on Performance and Efficiency: a comparison of three controlling methods for a math game. Lecture Notes In Computer Science, [S.L.], p. 501-511, 2019. Springer International Publishing. http://dx.doi.org/10.1007/978-3-030-34350-7 48.

MICHAEL, D. R. \& CHEN, S. L. Serious Games: Games That Educate, Train and Inform. Boston: Cengage Learning . 2006.

MOHER, David; STEWART, Lesley; SHEKELLE, Paul. All in the family: systematic reviews, rapid reviews, scoping reviews, realist reviews, and more. 2015. Systematic Reviews, vol. 4, no. 168

MAZIVIERO, Helio Fernando Gomes. Jogos digitais no ensino de matemática - o desenvolvimento de um instrumento de apoio ao diagnóstico das concepções dos alunos sobre diferentes representações dos números. 2014. 119 f. Dissertação (Mestrado) - Curso de Matemática, Faculdade de Ciências, Universidade Estadual Paulista., Bauru, 2014.

NINAUS, Manuel; KIILI, Kristian; MCMULLEN, Jake; MOELLER, Korbinian. Assessing fraction knowledge by a digital game. Computers In Human Behavior, [S.L.], v. 70, p. 197-206, maio 2017. Elsevier BV. http://dx.doi.org/10.1016/j.chb.2017.01.004.

PEDDYCORD-LIU, Zhongxiu; CODY, Christa; KESSLER, Sarah; BARNES, Tiffany; LYNCH, Collin F.; RUTHERFORD, Teomara. Using Serious Game Analytics to Inform Digital Curricular Sequencing. Proceedings Of The Annual Symposium On Computer-Human Interaction In Play, [S.L.], p. 195-204, 15 out. 2017. ACM. http://dx.doi.org/10.1145/3116595.3116620.

PEDDYCORD-LIU, Zhongxiu; HARRED, Rachel; KARAMARKOVICH, Sarah; BARNES, Tiffany; LYNCH, Collin; RUTHERFORD, Teomara. Learning Curve Analysis in a Large-Scale, Drill-and-Practice Serious Math Game: where is learning support needed?. Lecture Notes In Computer Science, [S.L.], p. 436-449, 2018. Springer International Publishing. http://dx.doi.org/10.1007/978-3-319-93843-1_32.

RUIPÉREZ-VALIENTE, José A.; KIM, Yoon Jeon. Effects of solo vs. collaborative play in a digital learning game on geometry: results from a k12 experiment. Computers \& Education, [S.L.], v. 159, p. 104008, dez. 2020. Elsevier BV. http://dx.doi.org/10.1016/j.compedu.2020.104008. 